\title{
Retraction Note to: Resibufogenin suppresses tumor growth and inhibits glycolysis in ovarian cancer by modulating PIM1
}

\author{
Qian $\mathrm{Li}^{1} \cdot$ Chuanwu Jiang ${ }^{2,3} \cdot$ Yan Wang ${ }^{2} \cdot$ Minghua Wei $^{2} \cdot$ Huijin Zheng ${ }^{2} \cdot Y_{a n q i} \mathrm{Xu}^{2} \cdot \mathrm{Xuegang} \mathrm{Xu}^{4} \cdot \mathrm{Fengyu} \mathrm{Jia}^{3} \cdot$ \\ Kai Liu ${ }^{3} \cdot$ Gang Sun ${ }^{3} \cdot$ Jianhua Zang ${ }^{5} \cdot$ Ping $\mathrm{Mo}^{6}$
}

Published online: 26 November 2020

(C) Springer-Verlag GmbH Germany, part of Springer Nature 2020

\section{Retraction Note to: Naunyn-Schmiedeberg's Archives of Pharmacology (2019) 392:1477-1489 https://doi.org/10.1007/s00210-019-01687-2}

The Editor in Chief retracted this article (1) because of significant concerns regarding a number of Figures presented in this work, which question the integrity of the data. Specifically: Figure 2B contains images that appear to be the same or nearly the same as those published previously in a number of unrelated publications $(2,3,4$ - now retracted).

Figure $3 \mathrm{C}$ and $5 \mathrm{C}$ contains a number of histopathology images which appear to have been previously published (5 - now retracted).

The authors were unable to provide raw data.

Author Ping Mo agreed to retraction but has not stated if they agree to this retraction notice. The remaining authors did not respond to any correspondence from the editor or publisher about this retraction.

\section{References}

Bo L, Shou Y (2018) Amentoflavone suppresses hepatocellu-lar carcinoma by repressing hexokinase 2 expression through inhibiting JAK2/ STAT3 signaling. Biomed Pharmacother 107:243-253.

Li Q, Jiang C, Wang Y (2019) Resibufogenin suppresses tumor growth and inhibits glycolysis in ovarian cancer by modulating PIM1. Naunyn-Schmiedeberg's Arch Pharmacol 392:1477-1489. https:// doi.org/10.1007/s00210-019-01687-2

Qiang W, Yi Yan J, Zhang P, Guo Yuqing X, Yong W, Fawei Q, Qingyun Z (2018) Physcion 8-O- $\beta$-glucopyranoside inhibits clearcell renal cell carcinoma bydownregulating hexokinase II and inhibiting glycolysis. Biomed Pharmacother 104:28-35.

Xiangyang D, Meihua W, Tao Z, Jiapei Y (2019) Physcion 8-O- $\beta$ glucopyranoside inhibits testicular germ cell tumors through regulating microRNA-199a. Anat Rec. https://doi.org/10.1002/ar.24324

Xiaoping P, Chen W, Yan L, Lida Z, Ti Z (2018) Protective autophagy induced by physcion suppresses hepato-cellular carcinoma cell metastasis by inactivating the JAK2/STAT3 Axis. Life Sci 214:124 135.

Publisher's note Springer Nature remains neutral with regard to jurisdictional claims in published maps and institutional affiliations.
The online version of the original article can be found at https://doi.org/10. 1007/s00210-019-01687-2

Jianhua Zang

jianhuazangqdhc@126.com

Ping Mo

pingmoqdhc@126.com

1 Department of Gynecology, Jining No. 1 People's Hospital, Jining, Shandong, China

2 Department of Interventional Radiology, Qingdao Hospital of Traditional Chinese Medicine, Qingdao, Shandong, China
Department of Interventional Radiology, Jinan Military General Hospital, Jinan, Shandong, China

4 Department of Interventional Radiology, Jining No. 2 People's Hospital, Jining, Shandong, China

5 Department of Oncology, Qingdao Hospital of Traditional Chinese Medicine, Renmin Road 4, Qingdao 266000, Shandong, China

6 Department of Gynecology, Qingdao Hospital of Traditional Chinese Medicine, Renmin Road 4, Qingdao 266000, Shandong, China 